\title{
UNDERDENSE PLASMA LENS EXPERIMENT AT THE UCLA NEPTUNE LABORATORY*
}

\author{
$\underline{\text { H. Suk }}^{\dagger}$, C.E. Clayton, G. Hairapetian ${ }^{\ddagger}$, C. Joshi, M. Loh, P. Muggli, \\ R. Narang, C. Pellegrini, J.B. Rosenzweig, UCLA, Los Angeles, CA 90095 \\ T.C. Katsouleas, USC, Los Angeles, CA 90089
}

\begin{abstract}
An underdense plasma-lens experiment is planned at the UCLA Neptune Laboratory. For this experiment, $\mathrm{a} \mathrm{LaB}_{6-}$ based discharge plasma source was developed and tested. Test results of the plasma source show that it can provide satisfactory argon plasma parameters for underdense plasma lens experiments, i.e., a density in low $10^{12} \mathrm{~cm}^{-3}$ range and a thickness of a few $\mathrm{cm}$. In the plasma chamber a YAG slab and a Cherenkov radiator are placed for electron beam diagnostics so that both time-integrated and time-resolved information will be obtained and compared with the MAGIC code ( 2 and 1/2 dimensional particle-incell) simulations. In this paper, the planned experiment including test results of the plasma source, diagnostics and MAGIC simulation results is presented.
\end{abstract}

\section{INTRODUCTION}

The UCLA Neptune beamline was commissioned recently and measurements of basic beam parameters are under way[1]. After initial beam characterizations a series of experiments will be conducted. One of them is the underdense plasma lens experiment, where the beam density is larger than the plasma density. Although some thick plasma lens experiments in underdense and overdense regimes were done before, a thin (in the sense that the plasma thickness is smaller than the focal length) underdense plasma lens has not been explored experimentally yet. Thus, a $\mathrm{LaB}_{6}$-based plasma source was developed and tested for this experiment at the UCLA Neptune Laboratory. In the ongoing plasma lens experiment, relatively long electron beams will be used so that the head part of the beam is used to produce a nearly uniform ion channel by expelling out the plasma electrons, while the tail part of the beam is focused due to the plasma lens effect of the ion channel.

\section{PLANNED EXPERIMENT AND PLASMA SOURCE TEST}

The plasma lens experiment will be done in the Neptune Laboratory, which consists of the beamline, drive laser for

\footnotetext{
* Work supported by the U.S. Department of Energy and NSF under contract numbers DE-FG03-92ER40693 and ECS 9617089.

† Email: suk@stout.physics.ucla.edu

$\ddagger$ Now working at HRL Laboratories
}

the photocathode RF gun, RF system, plasma source, etc. Details of each part are shown elsewhere [2,3] so that only brief descriptions are given here.

\subsection{Neptune Beamline}

The Neptune beamline consists of the new 1.625-cell photocathode RF gun, PWT linac, chicane, focusing and deflection magnets, etc. The photocathode RF gun ( $\mathrm{f}=2.856$ $\mathrm{GHz}$ ) has a copper cathode and it is illuminated by $266-\mathrm{nm}$ UV laser beams for photoelectron generation. In the RF gun, electron beams are accelerated to about $4.5 \mathrm{MeV}$, and then the beams are injected into the PWT linac with 7 and 2 half cells for further acceleration. In this way, the beams are accelerated up to about $16 \mathrm{MeV}$.

\subsection{Photocathode Drive Laser}

To generate photoelectrons from the copper cathode of the RF gun, high power UV laser beams are required. As will be shown in Sec. 3, the plasma lens experiment needs electron beams with about 25 ps (FWHM) or longer duration. To generate this kind of relatively long and high power UV laser beams, the 500-m-long optical fiber and the gratings in the present laser system are bypassed [4]. Hence, the photocathode drive laser system for the plasma lens experiment consists of the mode-locked Nd:YAG laser $(\lambda=1.064 \mu \mathrm{m}, 80 \mathrm{ps}$ FWHM $)$, Nd:glass regenerative amplifier, two BBO crystals for frequency quadrupling, etc. In this way, 266-nm UV beams with 25 to 30 ps duration can be generated.

\subsection{RF System}

The photocathode RF gun and PWT are powered by the same klystron (24 MW SLAC XK-5) and modulator. About $1 / 3$ of the klystron RF power is sent to the gun and about $2 / 3$ of the RF power is fed into the PWT linac. The RF pulse width can be changed in several $\mu$ s range by the modulator.

\subsection{Underdense Plasma Source}

The plasma source generates argon plasmas resulting from cathode-anode pulse discharges. Figure 1 shows the schematic of the plasma source. It consists of a 7-cmdiameter $\mathrm{LaB}_{6}$ cathode, tantalum hollow anode, beamplasma interaction chamber, solenoids for plasma confine- 
ment and guiding, etc. For thermal electron emission the $\mathrm{LaB}_{6}$ cathode is heated up to over $1300{ }^{\circ} \mathrm{C}$ by a $0.1-\Omega$ graphite heater, and a voltage pulse of about $100 \mathrm{~V}$ is applied between the cathode and anode for several ms. As a result, a discharge plasma is generated and the plasma is guided to flow towards the beam-plasma interaction chamber with the help of the confining magnetic field. At the entrance of the beam-plasma interaction chamber there is a sliding door to control plasma thickness.

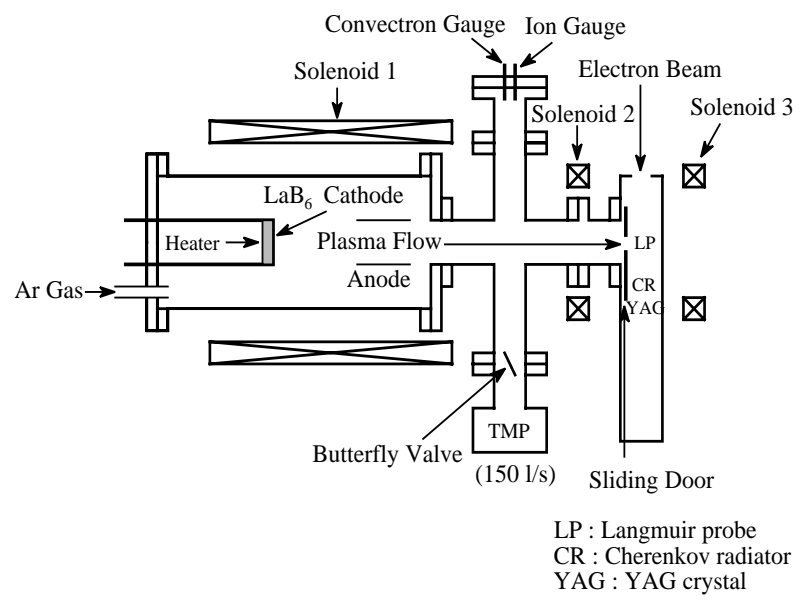

Figure 1: Schematic of the plasma source.

Plasma density in the beam-plasma interaction chamber depends on several parameters such as neutral argon pressure, discharge voltage, magnetic field strength and cathode temperature. To find the optimum condition the parameters were changed one by one and the plasma density was measured in the chamber with a cylindrical Langmuir probe. It turned out that the cathode temperature and magnetic field are two dominant factors. In other words, the plasma density increases significantly when the cathode temperature and magnetic field increases slightly.

Figure 2 shows plasma density profiles measured along the electron beam path for two different conditions. Figure 2(a) was obtained when the sliding door gap was $1.5 \mathrm{~cm}$, while Fig. 2(b) was obtained when the sliding door gap was $1 \mathrm{~cm}$. As expected, Fig. 2(b) profile has a smaller peak plasma density $\left(1.1 \times 10^{12} \mathrm{~cm}^{-3}\right)$ and narrower width (effective width $=2.9 \mathrm{~cm}$ ) compared to Fig. 2(a).

As shown in Fig. 1, high energy electron beams are designed to propagate in the plasma transversely and the beams will be focused downstream. To take timeintegrated beam images resulting from the plasma focusing, an undoped YAG slab with a thickness of $0.5 \mathrm{~mm}$ is installed in the beam-plasma interaction chamber. In addition, a Cherenkov radiator is placed in the chamber to obtain time-resolved information of the focused beams. Both the YAG slab and the Cherenkov radiator can be moved longitudinally along the beam path.
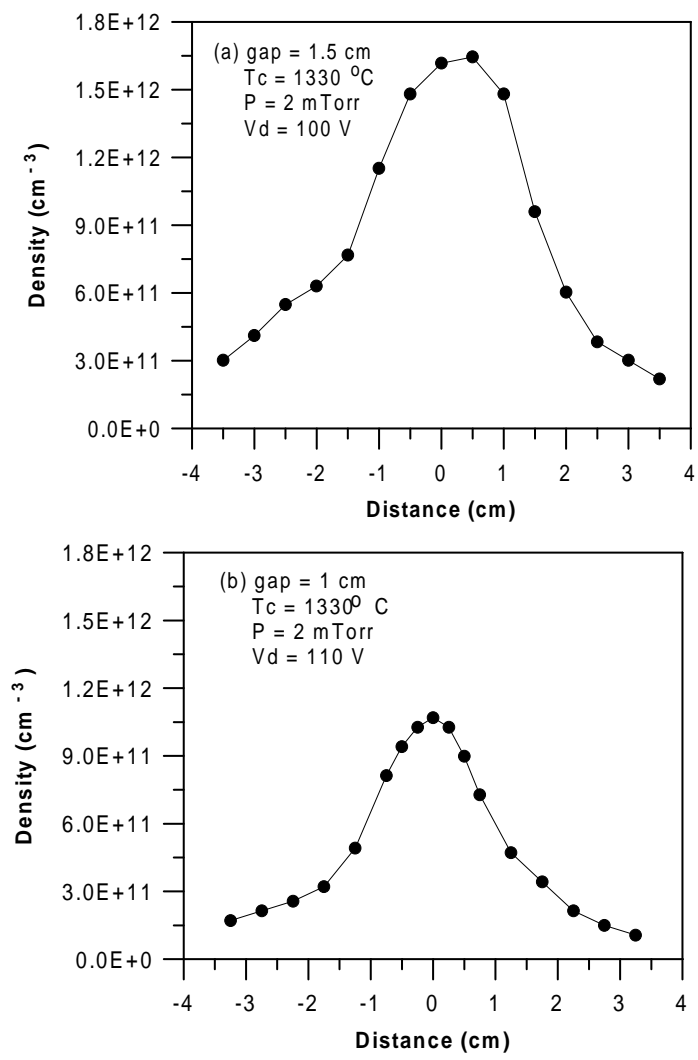

Figure 2: Longitudinal plasma density profiles for two different conditions.

\section{PARTICLE-IN-CELL CODE SIMULATION RESULTS}

To predict experimental results, computer simulations with a particle-in-cell code (MAGIC) have been performed. For this simulation the beam and plasma parameters in Table 1 were used.

Table 1: Beam and plasma parameters for simulations.

\begin{tabular}{|l|c|}
\hline beam energy & $16 \mathrm{MeV}$ \\
\hline charge per bunch & $4 \mathrm{nC}$ \\
\hline beam duration (FWHM) & $30 \mathrm{ps}$ \\
\hline normalized emittance & $10 \mathrm{~mm}-\mathrm{mrad}^{-3}$ \\
\hline beam density & $2.6 \times 10^{12} \mathrm{~cm}^{-3}$ \\
\hline beam radius at plasma entrance & $400 \mu \mathrm{m}$ \\
\hline plasma density & $1.1 \times 10^{12} \mathrm{~cm}^{-3}$ \\
\hline effective plasma thickness & $2.25 \mathrm{~cm}$ \\
\hline
\end{tabular}

Figure 3 shows beam particle trajectories. In this example, the beam density is 2.5 times larger than the plasma density and the beam rise time is also larger than the plasma oscillation time $\left(1 / \omega_{p}=17 \mathrm{ps}\right)$ so that the plasma electrons are almost adiabatically expelled from the beam path when the beam propagates in the plasma. Hence, after a couple of plasma oscillations a nearly uniform ion channel 


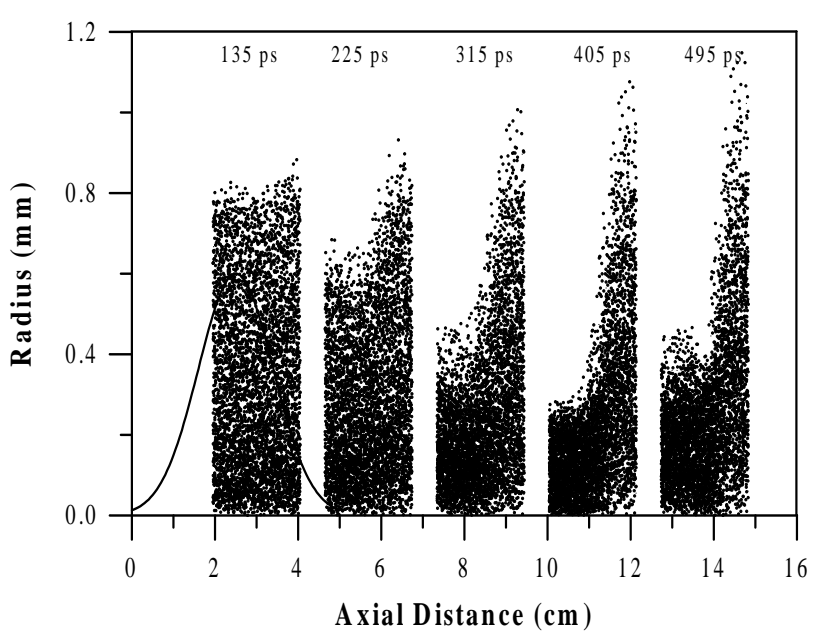

Figure 3: Electron beam trajectory. The solid line shows the plasma density profile with the parameters in Table 1.

is formed and the tail part of the beam is focused by the ion channel. This phenomenon is observed in Fig. 3. As shown in the figure, the head part of the beam is not focused and it continues to expand due to an emittance effect, while the tail part of the beam is tightly focused after about $2 / \omega_{p}$ and then it expands radially. Its focal length is observed to be about $9 \mathrm{~cm}$, which is in good agreement with the impulse focusing approximation [5].

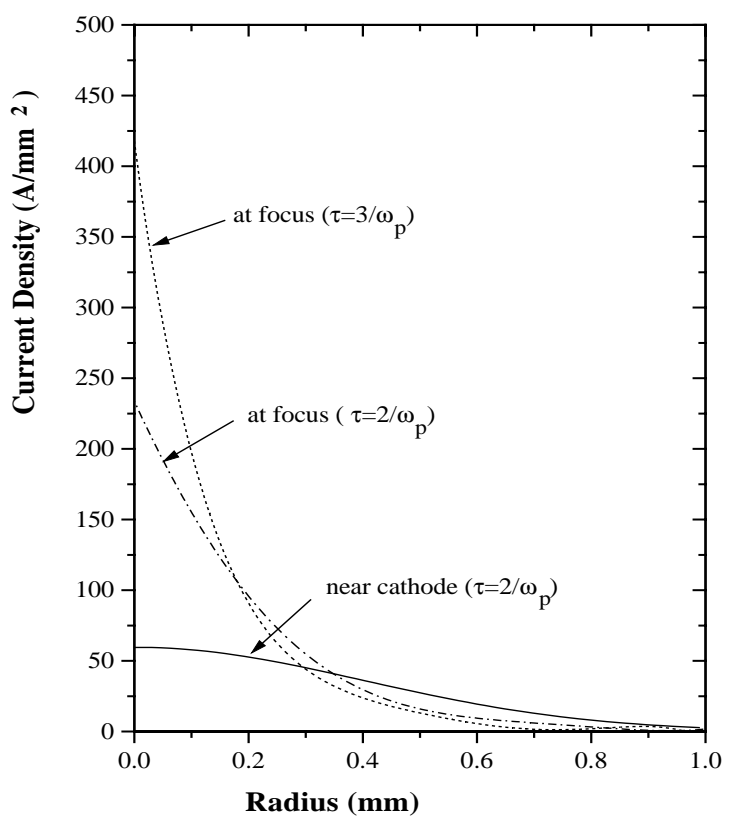

Figure 4: Beam current density profiles near cathode and at focus.
The plasma focusing will change a beam current density profile downstream. This is shown in Fig. 4, where the solid line represents the initial Gaussian density profile near the cathode and the other two dotted lines are profiles at the focus at different instants. It is shown that the beam is radially focused, but its current density profile at the focus is significantly deformed from the initial profile near the cathode. This is related with the highly dynamical process of an ion channel formation in the plasma. To reduce the nonlinear focusing effect the plasma and beam densities can be increased. In this case, the plasma oscillation period is smaller and the ion channel will be formed in a shorter time so that the tail part of the beam will experience a less nonlinear focusing. Then the density profile deformation will be mitigated.

\section{PRESENT STATUS AND CONCLUSIONS}

Since the test results of the plasma source were reported previously [3], a couple of problems in the plasma source have been improved. For example, some parts of the plasma source were too hot during operation in the past, but this problem was solved by adding an additional cooling structure. In addition, the solenoidal magnetic field for plasma confinement deflects electron beams transversely and this could be a problem for Cherenkov radiator-streak camera measurements. Hence, new solenoids with a smaller diameter and iron shielding were designed and the old solenoids were replaced by the new ones to minimize the beam deflection. Basically the plasma source is ready for experiments. Hence, after the ongoing measurements of basic beam parameters, the plasma source will be assembled with the Neptune beamline for the plasma lens experiment.

\section{REFERENCES}

[1] S. Aderson et al., "Commissioning of the Neptune Photoinjector", these proceedings.

[2] J.B. Rosenzweig et al., Nucl. Instr. and Meth. A 410, 437 (1998).

[3] H. Suk et al., "Test Results of the Plasma Source for Underdense Plasma Lens Experiments at the UCLA Neptune laboratory", to appear in Advanced Accelerator Concepts (AIP, New Nork, 1998).

[4] P. Davis, Plasma Lenses for Relativistic Electron Beams, $\mathrm{Ph}$. D. Dissertation, Department of Electrical Engineering, UCLA (1996).

[5] J.J. Su et al., Phys. Rev. A 41, 3321 (1990). 